\title{
Fachliches Metadatenmanagement mit einem semantischen Wiki
}

Um aktuellen Herausforderungen weltweiter Märkte begegnen zu können, brauchen Unternehmen ein einheitliches Verständnis ihrer Geschäftsobjekte. Einerseits sind regionale Spezifika zu unterstützen, um weltweit agieren und z.B. günstige Produktionsstandorte nutzen zu können. Andererseits sind einheitliche Datenstrukturen für unternehmensweite Analysen erforderlich, um z.B. globale Einkaufsstrategien umsetzen und vorteilhafte Einkaufskonditionen aushandeln zu können. Zusätzlich müssen Geschäftsobjekte neue Anforderungen seitens des Markts und regulierender Institutionen möglichst schnell abbilden - und das weltweit und konsistent. Ziel eines effektiven Managements von Geschäftsobjekt-Metadaten ist somit die Bereitstellung aktueller, detaillierter, flexibler und gleichzeitig unternehmensweit konsistenter Metadaten (z.B. technische und fachliche Spezifikationen, anwendungsspezifische Informationen zur korrekten Nutzung). Zur Unterstützung dieser Aufgabe stellt der Beitrag das Konzept eines fachlichen Metadatenkatalogs vor und diskutiert einen Wiki-basierten Prototyp, der gemeinsam mit dem Unternehmen Bayer Cropscience realisiert wurde. Die Evaluation des Prototyps zeigt, dass sich insbesondere semantische Wikis gut zur Realisierung eines fachlichen Metadatenkatalogs eignen.

\section{Inhaltsübersicht}

1 Metadaten zur Beschreibung von Geschäftsobjekten

2 Management fachlicher Metadaten mit Wikis

2.1 Wikis als Kollaborationsplattform in Unternehmen

2.2 Allgemeine Anforderungen an ein Business Data Dictionary
3 Ein Wiki als Business Data Dictionary bei Bayer Cropscience

3.1 Fachliches Metadatenmanagement bei Bayer Cropscience

3.2 Anforderungserhebung

3.3 Implementierung als semantisches Wiki

3.4 Evaluation und Szenariotests

4 Ergebnisse der Nutzung des Prototyps und weiterer Forschungsbedarf

5 Literatur

\section{Metadaten zur Beschreibung von Geschäftsobjekten}

Die Steuerung verteilter Unternehmen setzt ein klares und einheitliches Verständnis gemeinsam genutzter Daten voraus ("SingleSource-of-Truth«). Zudem basieren integrierte und automatisierte Geschäftsprozesse auf klaren und eindeutigen Definitionen von Geschäftsobjekten (z.B. Materialien, Kunden, Lieferanten, Stücklisten) [Kagermann \& Österle 2006, S. 230]. Diese meist unternehmensspezifische Semantik (z.B. technische und fachliche Spezifikationen, anwendungsspezifische Informationen zur korrekten Nutzung) wird durch Metadaten beschrieben [Burnett et al. 1999, S. 1213]. Metadaten hoher Qualität (insb. bezüglich Aktualität, Genauigkeit und Vollständigkeit) tragen zu einer reibungslosen Geschäftsprozessausführung bei, indem sie Fehler bei automatisierten Aktivitäten sowie lange Wartezeiten zwischen zwei Aktivitäten reduzieren. So ist beispielsweise in überbetrieblichen Geschäftsszenarien (z.B. Schienengüterverkehr auf Trassen unterschiedlicher nationaler Anbieter) ein unternehmensintern einheitliches Verständnis von Geschäftsobjekten (z.B. "Trasse" oder "Gefahrgut") Voraussetzung, um die unterschiedliche Semantik von Geschäftsobjekten 
verschiedener Geschäftspartner konsolidieren und in internen Geschäftsprozessen korrekt verarbeiten zu können.

Abbildung 1 erläutert die für den Beitrag wesentlichen Konzepte: Aktivitäten eines Geschäftsprozesses erzeugen und nutzen Geschäftsobjekte zur Leistungserstellung. Datenobjekte repräsentieren Geschäftsobjekte (z.B. einen Kunden) durch eine Auswahl bestimmter Objekteigenschaften (z.B. Name, Geburtsdatum) und deren Abbildung auf Datenattribute. Stammdaten sind Datenobjekte, deren Attributwerte sich im Gegensatz zu anderen Datenarten seltener ändern. Metadaten beschreiben technische und fachliche Eigenschaften (z.B. Datenstruktur, applikationsspezifische Nutzung) von Geschäftsobjekten [Burnett et al. 1999, S. 1213]. Ein fachlicher Metadatenkatalog (Business Data Dictionary - BDD) ist ein Werkzeug zur Unterstützung des Managements von technischen und fachlichen GeschäftsobjektMetadaten [Schmidt \& Otto 2008, S. 215].

Eine Barriere für unternehmensweit einheitliche und verständliche GeschäftsobjektMetadaten ist die Verteilung des benötigten Wissens über verschiedene Abteilungen eines Unternehmens [Newman \& Logan 2006, S. 3]. Keine zentrale Organisationseinheit ist in der Lage, die notwendigen Metadaten umfassend zu definieren und zu dokumentieren. Daher muss das fachliche Metadatenmanagement verteilt organisiert sein und Prinzipien der
Kooperation zwischen Fachexperten nutzen. Ein Werkzeug zur Unterstützung dieser verteilten Organisation sollte die kollaborative Erfassung des benötigten Wissens "vor Ort« ermöglichen, d.h. das Sammeln, Diskutieren und Konsolidieren verschiedener Aspekte eines Geschäftsobjekts (z.B. technische Merkmale, buchhalterische Attribute, regionale Spezifika). Die Ähnlichkeit des beschriebenen Prozesses mit der kollaborativen Erstellung und Pflege der Online-Enzyklopädie Wikipedia legt die Nutzung eines Wikis als unterstützendes Werkzeug nahe. Daher beschreibt der Beitrag das Konzept eines als Wiki implementierten BDD und diskutiert die Evaluation eines Prototyps im Unternehmen Bayer Cropscience.

\section{Management fachlicher Metadaten mit Wikis}

\subsection{Wikis als Kollaborationsplattform in Unternehmen}

Unterstützt durch den Erfolg von Wikipedia steigt das Interesse von Unternehmen an der Nutzung von Wikis für betriebliche Aufgaben [Bughin et al. 2008, S. 2]. Forschungsarbeiten zeigen, dass ein nachhaltiger Einsatz von Wikis in Unternehmen möglich ist [Majchrzak et al. 2006] und Wikis sich auch im betrieblichen Kontext für Spezifikationsprozesse erfolgreich einsetzen lassen [Wagner \& Majchrzak 2007]. Gleichwohl werden erhoffte Kollaborations-

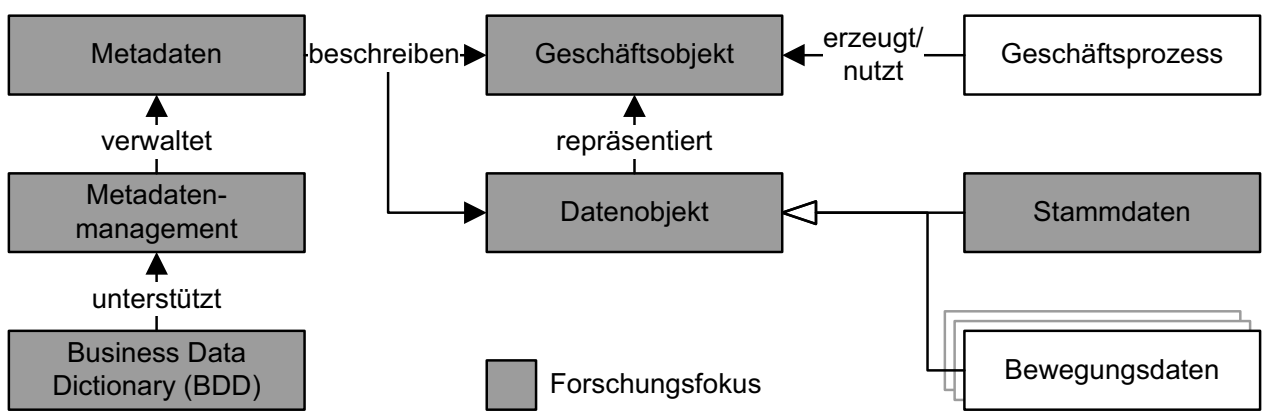

Abb. 1: Terminologischer Bezugsrahmen und Forschungsfokus 
effekte im betrieblichen Kontext nicht zwangsläufig erzielt [Majchrzak 2009, S. 18]. Die Entwicklung von Wikipedia zeigt außerdem, dass die unstrukturierte Verwaltung von Metadaten in einem Wiki zu Redundanzen und ineffizienter Nutzung führen kann [Krötzsch et al. 2007].

In Anlehnung an den hawaiianischen Begriff für "schnell« oder »rasch« ist ein Wiki eine Website, deren Inhalt jeder ohne großen Aufwand (eben "rasch «) anpassen und ergänzen kann [Cunningham 1995]. Je nach verwendeter Software unterscheidet sich die von einem Wiki angebotene Funktionalität, wobei zwei Funktionen praktisch immer vorhanden sind [Kane \& Fichman 2009, S. 2]: Die Funktion "open editing" ermöglicht das einfache Verändern des Wiki-Inhalts durch jeden Benutzer (u.U. auch anonym), "edit preservation « die Aufbewahrung aller Bearbeitungen und Versionen des Wiki-Inhalts. So kann beispielsweise ein "roll back" jeden Zustand des Wiki-Inhalts (z.B. zur Abwehr von Vandalismus) mit geringem Aufwand wiederherstellen.

Wie bereits angedeutet erfasst ein Wiki Metadaten weitgehend unstrukturiert. Die Gliederung des Wiki-Inhalts durch Artikel und Verknüpfungen zwischen Artikeln unterstützt einen menschlichen Nutzer, ermöglicht aber keine effiziente maschinelle Verarbeitung des Inhalts [Krötzsch et al. 2007]. Diesen Mangel adressieren semantische Wikis und bieten neben der normalen Wiki-Funktionalität zusätzliche Strukturierungsmöglichkeiten sowie darauf aufbauend erweiterte Navigationsunterstützung und Suchmechanismen [Schaffert et al. 2008]. Ein semantisches Wiki verarbeitet semantisch annotierte Verknüpfungen, d.h. zusätzliche Informationen zur Bedeutung einer Verknüpfung zwischen zwei Wiki-Artikeln, zu einer maschinenverarbeitbaren Struktur. Die Verknüpfung zwischen einem Artikel zu einer SAP-ERP-Transaktion und einem Artikel zu einem Geschäftsprozess kann beispielsweise mit der Information "genutzt in « annotiert werden. Das semantische Wiki verarbeitet diese Annota- tionen und kann auf Anfrage eine Liste aller in einem bestimmten Geschäftsprozess genutzten Transaktionen erzeugen. In einem normalen Wiki ist eine solche Auflistung nur durch manuelle Eingabe der einzelnen Listenelemente möglich.

Das Potenzial semantischer Wikis, insbesondere die Such- und Navigationsfunktionalität eines Wikis zu verbessern (z.B. Suche, Navigation), zeigt das Ergebnis eines Experiments [Hüner \& Otto 2009]. 62 Fachexperten verschiedener Unternehmen haben dabei vergleichbare Aufgaben (Suche und Pflege von Metadaten) in einem normalen und einem semantischen Wiki bearbeitet. Die Analyse zeigt, dass Nutzer im semantischen Wiki Metadaten schneller finden und die Benutzerfreundlichkeit besser bewerten (vgl. Abb. 2). Für die Pflege von Metadaten (d.h. Informationen ergänzen, Verknüpfungen annotieren) zeigt sich ein entgegengesetztes Ergebnis. Für die Interpretation der Resultate ist zu beachten, dass die verbesserte Nutzungsfunktionalität der entscheidende Faktor ist, um insbesondere die Akzeptanz des Wikis zu verbessern und so die Zahl der Nutzer zu erhöhen. Der in Abschnitt 3.3 vorgestellte Prototyp nutzt

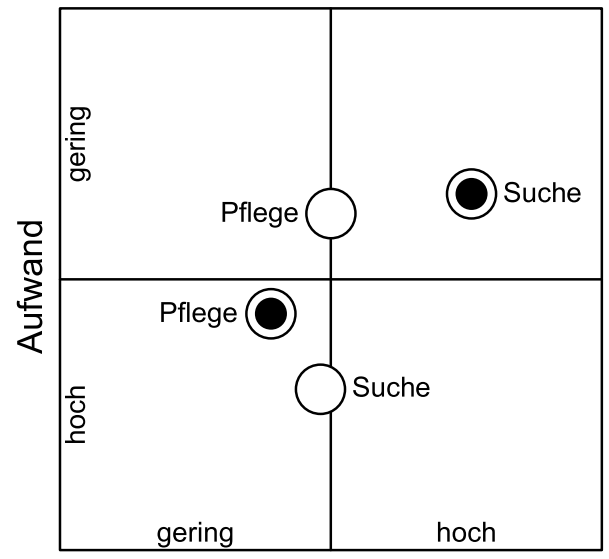

Benutzerfreundlichkeit

O Normales Wiki OSemantisches Wiki

Abb. 2: Ergebnis eines Experiments zu Potenzialen semantischer Wikis 
außerdem Formulare, die semantische Annotationen automatisch vornehmen und dadurch helfen, den höheren Pflegeaufwand auszugleichen.

\subsection{Allgemeine Anforderungen an ein Business Data Dictionary}

Das Kompetenzzentrum Corporate Data Quality (CC CDO, http://cdq.iwi.unisg.ch) ist Teil des Forschungsprogramms Business Engineering der Universität St. Gallen (www.iwi.unisg.ch/behsg). Es entwickelt seit 2006 gemeinsam mit verschiedenen Unternehmen Lösungen zur Unterstützung des Qualitätsmanagements konzernweit genutzter Daten. In diesem Forschungskontext wird auch untersucht, wie Wikis zur Unterstützung des fachlichen Metadatenmanagements genutzt werden können und welche zusätzlichen Anforderungen sich aus der betrieblichen Nutzung von Wikis für diese Aufgabe ergeben.

In Fokusgruppeninterviews hat das CC CDO zahlreiche Fallbeispiele diskutiert, in denen qualitativ schlechte Metadaten Geschäftsprobleme verursachen (vgl. Abschnitt 1). Konsens der Diskussionen war die Forderung eines Werkzeugs zur Unterstützung des fachlichen Metadatenmanagements, das einerseits Prozesse zur Definition und Pflege von Metadaten unterstützt und andererseits die Nutzung der Metadaten in möglichst vielen operativen Systemen (z.B. ERP-, CRM-System) und Abteilungen ermöglicht. Das Ergebnis eines Fokusgruppeninterviews mit 14 Fachexperten aus zehn Unternehmen ist eine Liste mit Anforderungen an ein solches BDD (vgl. Tab. 1).

\section{Ein Wiki als Business Data Dictionary bei Bayer CropScience}

\subsection{Fachliches Metadatenmanagement bei Bayer CropScience}

Die Bayer CropScience AG (ca. 18.300 Mitarbeiter, 6,4 Mrd. Euro Umsatz 2008) ist ein Geschäftsbereich der Bayer AG. Sie ist Marktführer im Bereich agrochemischer Produkte (z.B. Pflanzenschutz, Insektenbekämpfung, Saatgutzüchtung). Bayer CropScience führt im Zuge einer weltweiten Geschäftsprozessharmonisierung eine Konsolidierung von Stammdaten durch. Zukünftig soll ein zentrales System Materialund Geschäftspartnerstammdaten bereitstellen, die in den dann harmonisierten Geschäftsprozessen weltweit einheitlich verwendet werden. Aktuell sind Struktur und Bedeutung von Stammdaten teilweise regional unterschiedlich. So werden beispielsweise Logistikdaten zu Materialien wie Gewicht und Abmessungen in Europa anders gehandhabt als in Nord- und Südamerika bzw. Asien.

Bayer Cropscience nutzt bereits ein mit Lotus Notes realisiertes BDD. Dieses Master Data Handbook (MD-Handbuch) enthält Informationen zur Unterstützung des Stammdatenpflegeprozesses, beispielsweise zur korrekten Verwendung von Transaktionen und Feldern des unternehmensweit genutzten SAP-ERP-Systems. Eine Marktstudie zeigt, dass das aktuelle Marktangebot das Management technischer Metadaten nur teilweise und das Management fachlicher Metadaten fast gar nicht adressiert [White et al. 2009]. Daher sind Unternehmen bei der Unterstützung des fachlichen Metadatenmanagements auf Eigenentwicklungen (bei Bayer CropScience eine Datenbank in Lotus Notes) oder Kombinationen verschiedener Werkzeuge angewiesen.

\subsection{Anforderungserhebung}

Trotz der Verfügbarkeit des MD-Handbuchs blieb die Nutzeranzahl bei Bayer Cropscience gering (nur ca. 80 Nutzer von potenziell ca. 8.000). In Interviews wurden Defizite des Werkzeugs ermittelt und daraus Anforderungen an eine neue Lösung abgeleitet. Aufgrund der Diskussion kollaborativer Ansätze zur Unterstützung des fachlichen Metadatenmanagements im CC CDO (vgl. Abschnitt 2.2) hat Bayer CropScience entschieden, das MD-Handbuch durch ein Wikibasiertes BDD zu ersetzen und im Rahmen des 


\begin{tabular}{|c|c|}
\hline Anforderung & Erläuterung \\
\hline $\begin{array}{l}\text { A01. Flexible } \\
\text { Benutzerschnittstelle }\end{array}$ & $\begin{array}{l}\text { Ziel ist ein möglichst hoher Grad an Kollaboration, d.h. möglichst viele } \\
\text { aktive Nutzer aus möglichst vielen Bereichen eines Unternehmens. Die } \\
\text { Benutzerschnittstelle des BDD sollte daher individuell für verschiedene } \\
\text { Rollen mit unterschiedlichen Aufgaben und Fähigkeiten konfiguriert wer- } \\
\text { den können. }\end{array}$ \\
\hline $\begin{array}{l}\text { A02. Flexible } \\
\text { Metadatenstruktur }\end{array}$ & $\begin{array}{l}\text { Metadaten ändern sich im Zeitverlauf sowohl inhaltlich als auch strukturell } \\
\text { (z.B. zur Beschreibung neuer Datenattribute, durch die Einführung neuer } \\
\text { operativer Systeme, durch die Erweiterung um neue Sprachen). Die not- } \\
\text { wendigen Anpassungen müssen daher möglichst von jedem Nutzer vor- } \\
\text { genommen werden können, um insbesondere die Aktualität der } \\
\text { Metadaten sicherzustellen. }\end{array}$ \\
\hline $\begin{array}{l}\text { A03. Int } \\
\text { Portal u }\end{array}$ & $\begin{array}{l}\text { Um mögliche Vorgaben der IT-Strategie von Unternehmen zu erfüllen, } \\
\text { sollte das BDD in interne Informationssysteme wie ein Mitarbeiterportal } \\
\text { oder ein Intranet integriert werden können. }\end{array}$ \\
\hline $\begin{array}{l}\text { A04. Mehrsprachigkeit } \\
\text { von Software und } \\
\text { Metadaten }\end{array}$ & $\begin{array}{l}\text { Um einen hohen Grad an Kollaboration zu erzielen (vgl. A01. Flexible } \\
\text { Benutzerschnittstelle), müssen Metadaten in verschiedenen Sprachen } \\
\text { angeboten und erfasst werden können. Auch die Benutzerschnittstelle } \\
\text { des BDD sollte mehrsprachig sein. }\end{array}$ \\
\hline $\begin{array}{l}\text { isiertes } \\
\text { nat }\end{array}$ & $\begin{array}{l}\text { Die im BDD gepflegten Metadaten sollten möglichst sichtbar, d.h. in mög- } \\
\text { lichst vielen operativen Systemen verfügbar sein, um möglichst viele Mit- } \\
\text { arbeiter zur kollaborativen Pflege zu ermuntern. Mit einem } \\
\text { standardisierten Metadatenformat sind der Export der Metadaten aus dem } \\
\text { BDD und der Import der exportierten Metadaten in operative Systeme ein- } \\
\text { fach möglich. }\end{array}$ \\
\hline $\begin{array}{l}\text { A06. Unterstützung von } \\
\text { Nutzungs- und } \\
\text { Pflegeprozessen }\end{array}$ & $\begin{array}{l}\text { Klar definierte und dokumentierte Nutzungs- und Pflegeprozesse (z.B. } \\
\text { Unterstützung zur Suche im BDD, Workflows zur Aktualisierung oder Feh- } \\
\text { lermeldung) sichern die effiziente Nutzung des BDD. }\end{array}$ \\
\hline $\begin{array}{l}\text { A07. Metadaten- } \\
\text { verweise untereinander }\end{array}$ & $\begin{array}{l}\text { Lexika und Wörterbücher definieren einen Begriff in der Regel mithilfe } \\
\text { interner Verweise auf Definitionen anderer Begriffe, auf Glossare oder Bil- } \\
\text { der. Das BDD sollte solche Verweise ebenfalls ermöglichen, um redun- } \\
\text { dante Metadaten zu vermeiden und effiziente Navigation zu ermöglichen. }\end{array}$ \\
\hline $\begin{array}{l}\text { A08. Unterstützung von } \\
\text { Single-Sign-on }\end{array}$ & $\begin{array}{l}\text { Um mögliche Vorgaben der IT-Strategie von Unternehmen zu erfüllen, } \\
\text { sollte das BDD vorhandene Authentifizierungsverfahren integrieren kön- } \\
\text { nen und keine separate Authentifizierung erfordern. }\end{array}$ \\
\hline $\begin{array}{l}\text { A09. Verfügbarkeit der } \\
\text { Änderungshistorie }\end{array}$ & $\begin{array}{l}\text { Um Änderungen nachvollziehen und Fehler schnell korrigieren zu können, } \\
\text { sollte die Änderungshistorie der Metadaten gespeichert werden und die } \\
\text { einfache Wiederherstellung alter Zustände der Metadaten ermöglichen. }\end{array}$ \\
\hline
\end{tabular}

Tab. 1: Allgemeine Anforderungen an ein Business Data Dictionary

CC CDQ einen Prototyp zu entwickeln. Tabelle 2 zeigt die ermittelten Defizite des MD-Handbuchs und daraus abgeleitete Anforderungen. Außerdem wird für jede Anforderung die Umsetzung im Prototyp erläutert. Der Prototyp ist als semantisches Wiki mit der Software Semantic MediaWiki (SMW, www.semantic-mediawiki.org) implementiert, einer Erweiterung von
MediaWiki (www.mediawiki.org), der Wiki-Plattform von Wikipedia [Krötzsch et al. 2007].

Zwar wurden die in Tabelle 2 erläuterten Defizite unternehmensspezifisch für das MDHandbuch bei Bayer CropScience erhoben. Der Vergleich der abgeleiteten Anforderungen mit den allgemeinen Anforderungen aus Tabelle 1 zeigt jedoch, dass der für Bayer Cropscience 


\begin{tabular}{|c|c|c|}
\hline Defizit & $\begin{array}{l}\text { Abgeleitete } \\
\text { Anforderungen }\end{array}$ & Umsetzung im Prototyp \\
\hline \multirow[t]{2}{*}{$\begin{array}{l}\text { Fehlende } \\
\text { Einführung und } \\
\text { Dokumentation }\end{array}$} & $\begin{array}{l}\text { A10. Intuitiv zu bedienende } \\
\text { Benutzerschnittstelle (vgl. } \\
\text { A01) }\end{array}$ & $\begin{array}{l}\text { Basisfunktionalität von MediaWiki (z.B. Volltext- } \\
\text { suche) wird durch automatisch generierte Listen } \\
\text { (SMW-Funktionalität) ergänzt. Metadaten sind in } \\
\text { drei Aktualitätsklassen gegliedert. }\end{array}$ \\
\hline & $\begin{array}{l}\text { A11. Anleitungen und } \\
\text { Dokumentation im Wiki }\end{array}$ & $\begin{array}{l}\text { MediaWiki und SMW bieten eine umfangreiche } \\
\text { Funktionsdokumentation. BDD-spezifische Funkti- } \\
\text { onen und Konfigurationen sind noch nicht doku- } \\
\text { mentiert. }\end{array}$ \\
\hline \multirow[t]{3}{*}{$\begin{array}{l}\text { Geringe Qualität } \\
\text { des Inhalts }\end{array}$} & $\begin{array}{l}\text { A12. Feedback-Funktionen } \\
\text { (vgl. A06) }\end{array}$ & $\begin{array}{l}\text { Kommentieren von Artikeln ist Standardfunktionali- } \\
\text { tät von MediaWiki. }\end{array}$ \\
\hline & $\begin{array}{l}\text { A13. Einfache Identifikation } \\
\text { veralteter Metadaten (vgl. } \\
\text { A06) }\end{array}$ & $\begin{array}{l}\text { Durch Gliederung in drei Aktualitätsklassen kön- } \\
\text { nen veraltete Metadaten einfach identifiziert wer- } \\
\text { den. }\end{array}$ \\
\hline & $\begin{array}{l}\text { A14. Einfache Aktualisie- } \\
\text { rungsfunktionalität (vgl. } \\
\text { A06, A09) }\end{array}$ & $\begin{array}{l}\text { Formulare erleichtern die Dateneingabe und } \\
\text {-pflege. Die Realisierung der Validierungsfunktion } \\
\text { (vgl. Abschnitt 3.3) erforderte eine Erweiterung } \\
\text { (ca. } 40 \text { Zeilen PHP-Code). }\end{array}$ \\
\hline \multirow[t]{2}{*}{$\begin{array}{l}\text { Fehlende Mehr- } \\
\text { sprachigkeit }\end{array}$} & $\begin{array}{l}\text { A15. Mehrsprachige Benut- } \\
\text { zerschnittstelle (vgl. A04) }\end{array}$ & $\begin{array}{l}\text { Die Benutzerschnittstelle von MediaWiki und SMW } \\
\text { ist in zahlreichen Sprachen verfügbar. Nicht ver- } \\
\text { fügbare Sprachen können einfach ergänzt werden. }\end{array}$ \\
\hline & $\begin{array}{l}\text { A16. Unterstützung zur } \\
\text { Strukturierung mehrsprachi- } \\
\text { ger Inhalte (vgl. A06) }\end{array}$ & $\begin{array}{l}\text { Die Sprache des Wiki-Inhalts ist frei wählbar. } \\
\text { Relationen zur Verknüpfung verschiedener } \\
\text { Sprachversionen eines Artikels sind nicht enthal- } \\
\text { ten, können aber als semantische Annotationen } \\
\text { mit SMW einfach realisiert werden. }\end{array}$ \\
\hline $\begin{array}{l}\text { Unklare } \\
\text { Navigation }\end{array}$ & $\begin{array}{l}\text { A17. Übersichtliche Naviga- } \\
\text { tion (vgl. A06, A07) }\end{array}$ & $\begin{array}{l}\text { Die Navigation des Prototyps ist auf Basis von Nut- } \\
\text { zungsszenarien (vgl. Abschnitt 3.4) realisiert, die in } \\
\text { Interviews mit Nutzern identifiziert wurden. }\end{array}$ \\
\hline \multirow[t]{2}{*}{$\begin{array}{l}\text { Unstrukturierte } \\
\text { Suchergebnisse }\end{array}$} & $\begin{array}{l}\text { A18. Intuitive Suchfunktio- } \\
\text { nalität (vgl. A06) }\end{array}$ & $\begin{array}{l}\text { Die von MediaWiki bereitgestellte Volltextsuche } \\
\text { bietet die für Webseiten übliche Suchfunktionalität. }\end{array}$ \\
\hline & $\begin{array}{l}\text { A19. Vordefinierte Filter } \\
\text { (vgl. A06) }\end{array}$ & $\begin{array}{l}\text { Die Gliederung in drei Aktualitätsklassen stellt } \\
\text { einen einfachen vordefinierten Filter dar, der mit } \\
\text { Standardfunktionalität von SMW realisiert ist. }\end{array}$ \\
\hline $\begin{array}{l}\text { Unklare } \\
\text { Bezeichnungen } \\
\text { der Einträge }\end{array}$ & A20. Eindeutige Bezeichner & $\begin{array}{l}\text { Die Metadaten sind durch die Titel der Wiki-Artikel } \\
\text { eindeutig benannt. }\end{array}$ \\
\hline $\begin{array}{l}\text { Fehlende } \\
\text { Integration mit } \\
\text { SAP-System }\end{array}$ & $\begin{array}{l}\text { A21. Integration mit SAP- } \\
\text { System (vgl. A05) }\end{array}$ & $\begin{array}{l}\text { SMW kann die aus annotierten Verknüpfungen } \\
\text { generierte Metadatenstruktur in verschiedenen } \\
\text { Formaten (z.B. XML, CSV) exportieren. Bayer } \\
\text { CropScience plant, mit dieser Möglichkeit Meta- } \\
\text { daten aus dem BDD zu exportieren und im SAP- } \\
\text { System verfügbar zu machen. }\end{array}$ \\
\hline \multirow{2}{*}{$\begin{array}{l}\text { Fehlende Doku- } \\
\text { mentation von } \\
\text { Abhängigkeiten } \\
\text { zwischen } \\
\text { Feldern }\end{array}$} & \multicolumn{2}{|l|}{ vgl. A11 } \\
\hline & $\begin{array}{l}\text { A22. Unterstützung inhaltli- } \\
\text { cher Verknüpfungen (vgl. } \\
\text { A07) }\end{array}$ & $\begin{array}{l}\text { Verweise auf andere Wiki-Artikel sind Basisfunktio- } \\
\text { nalität von MediaWiki. }\end{array}$ \\
\hline
\end{tabular}




\begin{tabular}{|c|c|c|}
\hline Defizit & $\begin{array}{l}\text { Abgeleitete } \\
\text { Anforderungen }\end{array}$ & Umsetzung im Prototyp \\
\hline $\begin{array}{l}\text { Fehlende nut- } \\
\text { zerspezifische } \\
\text { Sichten }\end{array}$ & $\begin{array}{l}\text { A23. Unterstützung indivi- } \\
\text { dueller Wiki-Artikel (vgl. } \\
\text { A01) }\end{array}$ & $\begin{array}{l}\text { MediaWiki bietet vordefinierte Wiki-Artikel für regis- } \\
\text { trierte Nutzer. Spezielle Vorlagen für Metadaten- } \\
\text { pfleger sind vorgesehen. }\end{array}$ \\
\hline $\begin{array}{l}\text { Fehlende } \\
\text { Verweise zwi- } \\
\text { schen Objekten }\end{array}$ & A22 & \\
\hline
\end{tabular}

\section{Tab. 2: Defizite des MD-Handbuchs und abgeleitete Anforderungen an das Wiki}

entwickelte Prototyp auch allgemeine Anforderungen an ein BDD erfüllt. Ein wesentlicher Grund für die Realisierung des Prototyps als semantisches Wiki ist beispielsweise die fehlende Integration der Metadaten aus dem MD-Handbuch in das SAP-ERP-System von Bayer CropScience (vgl. A21). Mit dem standardisierten Metadatenformat von SMW (d.h. der aus annotierten Verweisen erzeugten Struktur) sind der Export der Metadaten aus dem Prototyp und der Import der exportierten Metadaten in das SAPERP-System einfach möglich, womit neben der spezifischen Anforderung A21 auch die allgemeine Anforderung A05 erfüllt ist.

\subsection{Implementierung als semantisches Wiki}

Als Erläuterung der zur Realisierung des Prototyps verwendeten Software SMW zeigt Abbildung za einen Wiki-Artikel mit Metadaten zu einer SAP-Transaktion, den sogenannten WikiText des Artikels mit Annotationen (Abb. 3b) und einen weiteren Wiki-Artikel, der Metadaten des Wikis automatisch anzeigt (Abb. 3c). Im WikiText der Metadaten zur Transaktion MMo1 ist die Verknüpfung zu Berthold Brauer (Pflegeverantwortlicher für die Transaktion) mit dem Attribut "Responsible" annotiert. SMW verarbeitet die annotierten Verknüpfungen und erstellt daraus eine maschinenverarbeitbare Struktur.

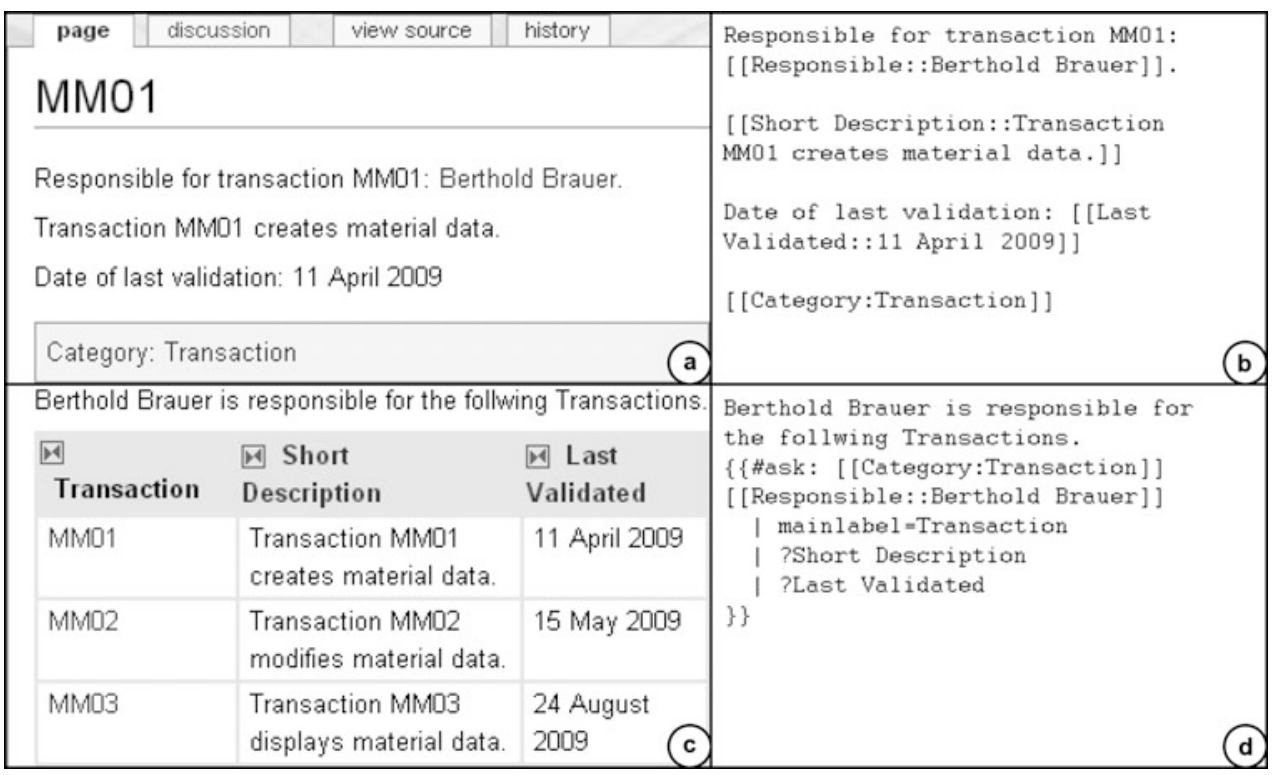

Abb. 3: Annotationen in einem semantischen Wiki 
Abbildung $3 c$ zeigt beispielsweise das Ergebnis einer Suchanfrage (Abb. 3d) zur Identifikation aller Transaktionen, für die Berthold Brauer verantwortlich ist. Die in der Liste dargestellten Daten können außerdem in einem standardisierten Metadatenformat (vgl. Ao5 in Tab. 1) exportiert und beispielsweise in ein SAP-ERP-System importiert werden (vgl. A21 in Tab. 2).

Abbildung 4 zeigt Ausschnitte der Benutzerschnittstelle des Prototyps. Dargestellt sind die Funktionalitäten, die im Rahmen eines Evaluationsszenarios (vgl. Abschnitt 3.4) von Mitarbeitern bei Bayer CropScience genutzt wurden. Die Startseite des Wikis bietet überschaubare Navigationshilfen (vgl. A17, A20). Abbildung 4a zeigt die vorgegebene Gliederung der Metadaten entsprechend ihrer Aktualität gemessen an der letzten Validierung durch den jeweils Verantwortlichen (vgl. A13). Alternativ zu dieser Navigation steht eine Volltextsuche zur Verfügung (vgl. A18). Die Sortierung der Metadaten nach ihrer Aktualität ist als Suchanfrage realisiert, ähnlich der in Abbildung 3d dargestellten Anfrage. Diese Funktionalität wäre mit unstrukturierten Metadaten in einem normalen Wiki nicht realisierbar.

Abbildung $4 b$ zeigt einen Ausschnitt eines Wiki-Artikels mit strukturierten Metadaten zu Verantwortlichkeiten und der letzten inhaltlichen Validierung. Die Farbe des Validierungsdatums ändert sich abhängig von der Aktualität des Datums: Artikel, die länger als sechs Monate nicht validiert wurden, werden z.B. gelb dargestellt (vgl. A13). Der dargestellte Reiter "validate" wird nur dann angezeigt, wenn der für einen Artikel Verantwortliche im Wiki angemeldet ist. Die Funktion des Reiters aktualisiert das Validierungsdatum (vgl. A14). Das Wiki ist so konfiguriert, dass Nutzer nur dann Schreibrechte haben, wenn sie angemeldet sind. Kommentieren ist für alle Nutzer (auch anonym) erlaubt. Die Notwendigkeit der Anmeldung von Metadatenpflegern ermöglicht die Bereitstellung individueller Benutzerartikel (vgl. A23) und vordefinierter Listen der Metadaten, an denen der jeweilige Pfleger beteiligt oder für die er verantwortlich ist.

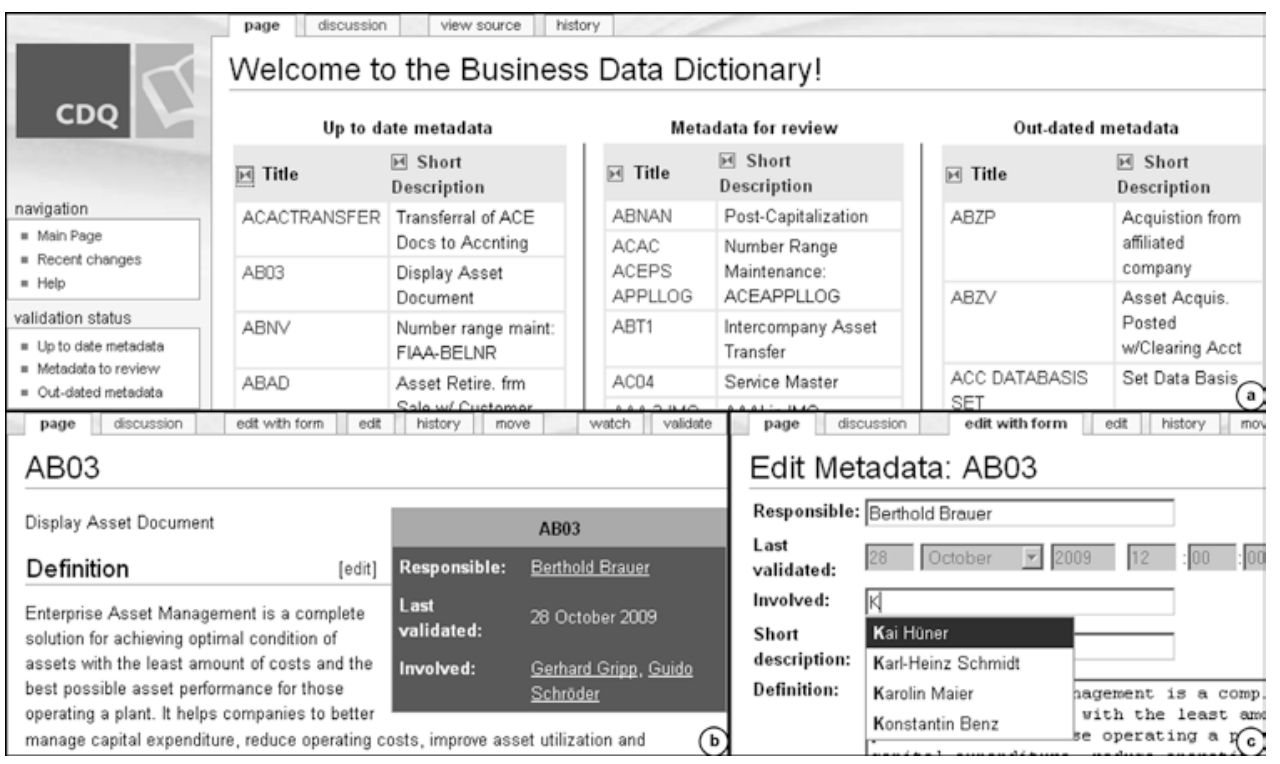

\section{Abb. 4: Ausschnitte der Benutzerschnittstelle des Prototyps}


Abbildung 4c zeigt ein Formular, das mithilfe der auf SMW basierenden Erweiterung Semantic Forms ${ }^{1}$ zur Pflege der Metadaten realisiert wurde. Durch Analyse des annotierten Wiki-Inhalts können mit der Erweiterung Auswahllisten zur Eingabeunterstützung generiert werden (vgl. Feld unter der Eingabe für »Involved« in Abb. 4C).

\subsection{Evaluation und Szenariotests}

Zur Evaluation des Prototyps bei Bayer Cropscience wurden aus den Ergebnissen der durchgeführten Interviews (vgl. Abschnitt 3.1) drei Nutzungsszenarien abgeleitet. Das Szenario "Metadaten pflegen " adressiert die Anforderungen A10, A12-A14, A17-A2O, A22 und A23. Es beschreibt die Meldung eines Metadatenfehlers durch einen Metadatennutzer und die Aktualisierung und Validierung der Metadaten durch einen Metadatenpfleger. Die anderen beiden Szenarien beschreiben die Nutzung von Metadaten im SAP-ERP-System und den Wechsel aus dem SAP-ERP-System in das BDD, um bestimmte Metadaten zu suchen.

Die zuvor interviewten Mitarbeiter von Bayer Cropscience (vgl. Abschnitt 3.1) haben den Prototyp in den drei Szenarien durch die Bearbeitung von gestellten Aufgaben (z.B. Suchen und Ändern bestimmter Metadaten) getestet und bewertet. Alle Tester kannten die alte Version des MD-Handbuchs und konnten so ein vergleichendes Urteil abgeben. Im Rahmen des Szenarios "Metadaten pflegen « waren die Tester aufgefordert,

- einen bestimmten Wiki-Artikel zu suchen,

- angegebene Metadaten zu prüfen,

- Fehler durch Kommentare im Wiki und per EMail an den jeweils Verantwortlichen zu kommunizieren,

- die durch die jeweils anderen Tester kommunizierten Fehler zu beheben und

- die vorgenommenen Änderungen zu validieren.

1. Website von Semantic Forms: www.mediawiki.org/wiki/Extension:Semantic_Forms.
Die Tester wurden nicht geschult und bekamen die Aufgaben schriftlich mitgeteilt. Aus technischer Sicht war die Nutzung des Prototyps ohne Probleme möglich. In Interviews haben die Tester nach Bearbeitung der Aufgaben die Benutzerfreundlichkeit bewertet. Alle Tester konnten alle Aufgaben bearbeiten. Das BDD ist somit auch von ungeschulten Mitarbeitern verwendbar. Alle Tester gaben an, dass sie der Prototyp sehr gut bei der Lösung der Aufgaben unterstützt hat. Im Vergleich zum MD-Handbuch wurden insbesondere die Einfachheit der Navigation, die flexiblen und verständlichen Listen, die Volltextsuche sowie die Verknüpfungen von Metadaten untereinander positiv bewertet. Die einzige negative Rückmeldung kritisierte die umständliche Verknüpfung externer Dateien mit dem alten MD-Handbuch war dies per »Drag \& Drop« möglich.

\section{Ergebnisse der Nutzung des Prototyps und weiterer Forschungsbedarf}

Der Beitrag hat das Konzept eines BDD und einen Wiki-basierten Prototyp beschrieben, der gemeinsam mit dem Unternehmen Bayer Cropscience realisiert wurde. Die Evaluation des Prototyps in Szenariotests zeigt, dass besonders die durch Verwendung eines semantischen Wikis mögliche Funktionalität die zuvor identifizierten Anforderungen an ein BDD erfüllt und die Benutzerfreundlichkeit erhöht. Wichtige Funktionen wie das Extrahieren von Metadaten mit bestimmten Eigenschaften (vgl. Abb. 4a) und der Export nur aktueller Metadaten oder von Metadaten, die eine bestimmte Anwendung betreffen, können mit einem normalen Wiki nicht realisiert werden. Um den positiven Kollaborationseffekt der Nutzung eines Wikis jedoch nicht durch zusätzliche Komplexität semantisch annotierter Metadaten zu verhindern, sollten die technischen und funktionalen Details eines semantischen Wikis vor Fachnutzern verborgen und nur zur Realisierung verbesserter 
Funktionalität genutzt werden. Ein Beispiel ist die Nutzung von Formularen, um bestimmte Metadaten (z.B. die Zuordnung von Verantwortlichen) strukturiert in das Wiki zu übernehmen und die notwendigen semantischen Annotationen automatisch vorzunehmen.

Weiterer Forschungsbedarf besteht in der Identifikation und Entwicklung weiterer Wikibasierter Funktionalität zur Unterstützung des fachlichen Metadatenmanagements und zur Integration von Metadaten in operative Systeme. Außerdem ist die Realisierbarkeit von Potenzialen wie

- Erhöhung der Nutzerzahl durch den Einsatz eines Wikis oder

- vollständigere und aktuellere Metadaten durch erhöhte Kollaboration

über einen längeren Zeitraum empirisch zu prüfen. Im CC CDO ermittelte Experteneinschätzungen (vgl. Abschnitt 2.2) deuten die Realisierbarkeit zwar an, weitere Untersuchungen müssen diese Vermutung jedoch bestätigen und zeigen, dass auch bisher nicht erreichte Nutzer das Konzept annehmen. Außerdem ist zu prüfen, inwieweit die Offenheit des Wiki-Konzepts die inhaltliche Qualität der Metadaten tatsächlich erhöht oder ob Änderungsrechte auf wenige Experten zu beschränken sind. Auch der positive Effekt der Integration von Metadaten in operative Systeme ist über einen längeren Zeitraum zu prüfen. Beispielsweise könnte die Bereitstellung der Metadaten direkt im ERP-System nach einer bestimmten Zeit wieder zu einer geringeren Nutzung des BDD und somit erneut $z u$ veralteten Metadaten führen, da das BDD und die Notwendigkeit der stetigen Metadatenpflege in Vergessenheit geraten.

\section{Literatur}

[Bughin et al. 2008] Bughin, J.; Chui, M.; Miller, A.: Building the Web 2.o Enterprise. McKinsey Global Survey Results. McKinsey Quarterly o.J. (2008), July, p. 1-10.
[Burnett et al. 1999] Burnett, K.; Ng, K. B.; Park, S.: A Comparison of the Two Traditions of Metadata Development. Journal of the American Society for Information Science 50 (1999), 13, p. 12091217.

[Cunningham 1995] Cunningham, W.: What Is Wiki. www.wiki.org/wiki.cgi?WhatlsWiki; Zugriff am: 23.11.2009.

[Hüner \& Otto 2009] Hüner, K. M.; Otto, B.: The Effect of Using a Semantic Wiki for Metadata Management: A Controlled Experiment. Proceedings of the 42nd Hawaii International Conference on System Sciences, 2009.

[Kagermann \& Österle 2006] Kagermann, H.; Österle, H.: Geschäftsmodelle 2010. Wie CEOs Unternehmen transformieren. Frankfurter Allgemeine Buch, 2006.

[Kane \& Fichman 2009] Kane, G. C.; Fichman, R. G.: The Shoemaker's Children: Using Wikis for Information Systems Teaching, Research, and Publication. Management Information Systems Quarterly 33 (2009) 1, p. 1-17.

[Krötzsch et al. 2007] Krötzsch, M.; Vrandei, D.; Völkel, M.; Haller, H.; Studer, R.: Semantic Wikipedia. Journal of Web Semantics 5 (2007), 4 , p. 251-261.

[Majchrzak 2009] Majchrzak, A.: Comment: Where is the Theory in Wikis? Management Information Systems Quarterly 33 (2009), 1, p. 18-20.

[Majchrzak et al. 2006] Majchrzak, A.; Wagner, C.; Yates, D.: Corporate Wiki Users: Results of a Survey. Proceedings of the 2006 International Symposium on Wikis, 2006, p. 99-104.

[Newman \& Logan 2006] Newman, D.; Logan, D.: Achieving Agility: How Enterprise Information Management Overcomes Information Silos. Gartner Research, Go0137817, Stamford, Connecticut, 2006.

[Schaffert et al. 2008] Schaffert, S.; Bry, F.; Baumeister, J.; Kiesel, M.: Semantic Wikis. IEEE Software 25 (2008), 4, p. 8-11.

[Schmidt \& Otto 2008] Schmidt, A.; Otto, B.: A Method for the Identification and Definition of Information Objects. Proceedings of the 13th International Conference on Information Quality, 2008, p. 214-228. 
[Wagner \& Majchrzak 2007] Wagner, C.; Majchrz-

Dipl.-Inform. Kai M. Hüner $a k, A_{\text {.: }}$ Enabling Customer-Centricity Using WiDr.-Ing. Boris Otto kis and the Wiki Way. Journal of Management Information Systems 23 (2007), 3, p. 17-43.

[White et al. 2009] White, A.; Radcliffe, J.; Wilson, D.: Vendor Guide: Master Data Management, 2009. Gartner Research, Goo164891, Stamford, Connecticut, 2009.

Prof. Dr. Hubert Österle Universität St. Gallen Institut für Wirtschaftsinformatik Müller-Friedberg-Str. 8

$\mathrm{CH}-9000$ St. Gallen $\{$ kai.huener, boris.otto, hubert.oesterle\}@unisg.ch www.unisg.ch

Berthold Brauer Bayer Cropscience AG Organization \& Information Services Alfred-Nobel-Str. 50 40789 Monheim am Rhein berthold.brauer@ bayercropscience.com www.bayercropscience.com 